\title{
COMPARISON BETWEEN THE KOBAYASHI AND CARATHÉODORY DISTANCES ON STRONGLY PSEUDOCONVEX BOUNDED DOMAINS IN $\mathbf{C}^{n}$
}

\author{
SERGIO VENTURINI
}

(Communicated by Irwin Kra)

\begin{abstract}
In this paper we prove that the ratio between the Caratheodory distance and the Kobayashi distance in a strongly pseudoconvex bounded domain in $\mathbf{C}^{n}$ is arbitrarily close to 1 whenever at least one of the points is sufficiently near the boundary.
\end{abstract}

\section{INTRODUCTION}

For every bounded domain $D \Subset \mathbf{C}^{n}$, we denote by $k_{D}$ and $c_{D}$ respectively the Kobayashi and Carathéodory distances and by $\kappa_{D}$ and $\gamma_{D}$ the corresponding infinitesimal metrics (cf. [8], [11], [5]).

It is a well-known result of Lempert that on convex domains the Kobayashi and Carathéodory distances (resp metrics) coincide ([9], [10]).

For an arbitrary domain an elementary argument yields $c_{D} \leq k_{D}$ and $\gamma_{D} \leq \kappa_{D}$.

The main result of this paper is the following theorem:

Theorem 1. Let $D \Subset \mathbf{C}^{n}$ be a strongly pseudoconvex bounded domain with $C^{2}$ boundary. For every $\varepsilon>0$ there exists a compact set $K \Subset D$, depending only on $\varepsilon$, such that if $z \in D \backslash K$ then for every $w \in D$ we have

$$
k_{D}(z, w) \leq(1+\varepsilon) c_{D}(z, w) .
$$

The main tools for the proof are the following:

(i) the results of Lempert on the coincidence of the Kobayashi and Carathéodory distances and infinitesimal metrics on convex domains;

(ii) the results of Graham on the localization of the Kobayashi and Carathéodory infinitesimal metric near the boundary;

(iii) some boundary estimates for the Kobayashi and Carathéodory distances obtained by Voormor, Forstneric, Rosay, Fadlalla and Abate;

(iv) an embedding theorem of Fornaess.

Received by the editors November 14, 1988, and in revised form March 2, 1989. 1980 Mathematics Subject Classification (1985 Revision). Primary 32H15. 
Throughout the paper we also prove some auxiliary results which are of independent interest (cf. Propositions 3 and 4).

\section{THE PROOF}

Throughout the paper $D$ stands for a strongly pseudoconvex bounded domain with $C^{2}$ boundary in $\mathbf{C}^{n}$. First we recall the localization results of Graham for infinitesimal metrics (cf. [6]).

Proposition 1. Let $x \in \partial D$ and let $U$ be a neighborhood of $x$ with $D \cap U$ connected. Then

$$
\lim _{z \rightarrow x} \frac{\kappa_{D \cap U}(z, v)}{\kappa_{D}(z, v)}=\lim _{z \rightarrow x} \frac{\gamma_{D \cap U}(z, v)}{\gamma_{D}(z, v)}=1,
$$

uniformly for $v \in \mathbf{C}^{n} \backslash\{0\}$.

Proposition 1 yields

$$
\lim _{z \rightarrow \partial D} \frac{\kappa_{D}(z, v)}{\gamma_{D}(z, v)}=1
$$

uniformly for $v \in \mathbf{C}^{n} \backslash\{0\}$. Indeed, if (3) is not true, then there exist $\varepsilon_{0}>0$ and sequences $\left\{z_{\nu}\right\} \subset D,\left\{v_{\nu}\right\} \subset \mathbf{C}^{n} \backslash\{0\}$ such that $z_{\nu} \rightarrow \partial D$ and

$$
\kappa_{D}\left(z_{\nu}, v_{\nu}\right) \geq\left(1+\varepsilon_{0}\right) \gamma_{D}\left(z_{\nu}, v_{\nu}\right) \text {. }
$$

By the compactness of $\partial D$ we can suppose that there exists $x \in \partial D$ such that $z_{\nu} \rightarrow x$. Let $U$ be a neighborhood of $x$ such that $D \cap U$ is biholomorphic to a convex set. Then we have $\kappa_{D \cap U}=\gamma_{D \cap U}$ and therefore by (2)

$$
\lim _{\nu \rightarrow+\infty} \frac{\kappa_{D}\left(z_{\nu}, v_{\nu}\right)}{\gamma_{D}\left(z_{\nu}, v_{\nu}\right)}=\lim _{\nu \rightarrow+\infty} \frac{\kappa_{D}\left(z_{\nu}, v_{\nu}\right)}{\kappa_{D \cap U}\left(z_{\nu}, v_{\nu}\right)} \frac{\gamma_{D \cap U}\left(z_{\nu}, v_{\nu}\right)}{\gamma_{D}\left(z_{\nu}, v_{\nu}\right)}=1,
$$

which contradicts (4).

In the following proposition we summarize the boundary estimates for the Kobayashi and Carathéodory distances that we need.

Proposition 2. Denote by $\|z\|$ and by $d(z, \partial D)$ respectively the Euclidean norm of $z$ and the distance of $z$ from the boundary of $D$. Then

(i) for every choice of $z \in D$ and $x \in \partial D$, there exist $C_{1}, C_{2} \in \mathbf{R}$ and $a$ neighborhood $U$ of $x$ such that for all $w \in D \cap U$ we have

(5) $-\frac{1}{2} \log d(w, \partial D)+C_{1} \leq c_{D}(z, w) \leq k_{D}(z, w) \leq-\frac{1}{2} \log d(w, \partial D)+C_{2}$;

(ii) for every choice of $x$ and $y$ in $\partial D$ with $x \neq y$ there exist neighborhoods $U$ and $V$ respectively of $x$ and $y$ and constants $C_{1}, C_{2} \in \mathbf{R}$ such that for all $z \in D \cap U$ and $w \in D \cap V$ we have

(6) $-\frac{1}{2} \log d(z, \partial D)-\frac{1}{2} \log d(w, \partial D)+C_{1} \leq c_{D}(z, w) \leq k_{D}(z, w)$

$$
\leq-\frac{1}{2} \log d(z, \partial D)-\frac{1}{2} \log d(w, \partial D)+C_{2} \text {; }
$$


(iii) for every $x \in \partial D$ there exists a neighborhood $U$ of $x$ and a constant $C \in \mathbf{R}$ such that for every choice of $z_{1}$ and $z_{2}$ in $D \cap U$ we have

(7) $k_{D}\left(z_{1}, z_{2}\right) \leq \sum_{j=1}^{2}-\frac{1}{2} \log d\left(z_{j}, \partial D\right)+\sum_{j=1}^{2} \frac{1}{2} \log \left(d\left(z_{j}, \partial D\right)+\left\|z_{1}-z_{2}\right\|\right)+C$.

The proofs of (5), (6) and (7) are in [13], [4], [1] and [2]. Now we need an analog of Proposition 1 for the Kobayashi distance; the idea is to use (2) and the fact that the Kobayashi distance is the integrated form of the corresponding infinitesimal metric. The difficulty here is to have a control on the paths of integration. This is achieved with the help of the following geometric lemma.

Lemma 1. Let $x \in \partial D$ and let $\left\{z_{\nu}\right\},\left\{w_{\nu}\right\},\left\{t_{\nu}\right\} \subset D$ be three sequences. Suppose that

$$
\lim _{\nu \rightarrow+\infty} z_{\nu}=\lim _{\nu \rightarrow+\infty} w_{\nu}=x
$$

and there exists a constant $C \geq 0$ such that for every $\nu \in \mathbf{N}$ we have

$$
k_{D}\left(z_{\nu}, t_{\nu}\right)+k_{D}\left(t_{\nu}, w_{\nu}\right) \leq k_{D}\left(z_{\nu}, w_{\nu}\right)+C .
$$

Then

$$
\lim _{\nu \rightarrow+\infty} t_{\nu}=x
$$

Proof. Suppose that the sequence $t_{\nu}$ does not converge to $x$. Since $D$ is bounded we can suppose that $t_{\nu} \rightarrow y$, with $y \in \bar{D}$ and $x \neq y$. First suppose that $y \in D$. Then, for suitable constants $C_{1}$ and $C_{2}$, by (5) we have

$$
\begin{aligned}
-\frac{1}{2} \log d\left(z_{\nu}, \partial D\right) & -\frac{1}{2} \log d\left(w_{\nu}, \partial D\right)+C_{1} \leq k_{D}\left(z_{\nu}, y\right)+k_{D}\left(w_{\nu}, y\right) \\
& \leq k_{D}\left(z_{\nu}, t_{\nu}\right)+k_{D}\left(w_{\nu}, t_{\nu}\right)+2 k_{D}\left(t_{\nu}, y\right) \\
& \leq k_{D}\left(z_{\nu}, w_{\nu}\right)+C+2 k_{D}\left(t_{\nu}, y\right) \\
& \leq k_{D}\left(z_{\nu}, w_{\nu}\right)+C_{2},
\end{aligned}
$$

since the sequence $k_{D}\left(t_{\nu}, y\right)$ is bounded.

If $y \in \partial D$ then by (6) for some $C_{1}$ we have

$$
\begin{aligned}
& -\frac{1}{2} \log d\left(z_{\nu}, \partial D\right)-\frac{1}{2} \log d\left(t_{\nu}, \partial D\right)+C_{1} \leq k_{D}\left(z_{\nu}, t_{\nu}\right), \\
& -\frac{1}{2} \log d\left(w_{\nu}, \partial D\right)-\frac{1}{2} \log d\left(t_{\nu}, \partial D\right)+C_{1} \leq k_{D}\left(w_{\nu}, t_{\nu}\right) .
\end{aligned}
$$

Summing we obtain

$$
\begin{aligned}
-\frac{1}{2} \log d\left(z_{\nu}, \partial D\right) & -\frac{1}{2} \log d\left(w_{\nu}, \partial D\right)-\log d\left(t_{\nu}, \partial D\right)+2 C_{1} \leq \\
& \leq k_{D}\left(z_{\nu}, t_{\nu}\right)+k_{D}\left(w_{\nu}, t_{\nu}\right) \leq k_{D}\left(z_{\nu}, w_{\nu}\right)+C .
\end{aligned}
$$

In both cases, combining the inequalities obtained with the estimate (7) for $k_{D}\left(z_{\nu}, w_{\nu}\right)$ we get a contradiction, Q.E.D.

Remark. A similar argument, and the estimates in [4] for the boundary behavior of the Kobayashi distance, yield the same result assuming the strongly pseudoconvexity of $\partial D$ only at $x$.

Remark. The lemma is clearly true also replacing the Kobayashi with the Carathéodory distance. 
Proposition 3. Let $x \in \partial D$ and let $U$ be a neighborhood of $x$ in $\mathbf{C}^{n}$ with $D \cap U$ connected. Then

$$
\lim _{z \neq w \rightarrow x} \frac{k_{D \cap U}(z, w)}{k_{D}(z, w)}=1 .
$$

Proof. Let $x \in \partial D$ and $U$ be a neighborhood of $x$ with $D \cap U$ connected. Let $\left\{z_{\nu}\right\},\left\{w_{\nu}\right\} \subset D \cap U$ be two arbitrary sequences converging to $x$ with $z_{\nu} \neq w_{\nu}$ and let $\varepsilon>0$. By Proposition 1 there exists a neighborhood $V \subset U$ of $x$ such that for every $z \in D \cap V$ and $v \in \mathbf{C}^{n}$ we have

$$
\kappa_{D \cap U}(z, v) \leq(1+\varepsilon) \kappa_{D}(z, v) .
$$

Let $\varepsilon_{\nu}=\min \left\{\varepsilon, k_{D}\left(z_{\nu}, w_{\nu}\right)^{-1}\right\}$. Since the Kobayashi distance is the integrated form of the Kobayashi infinitesimal metric (cf. [11] for a precise statement and [12] for a new proof) we can find $C^{\infty}$ arcs $\sigma_{\nu}:[0,1] \rightarrow D$ joining $z_{\nu}$ with $w_{\nu}$ such that

$$
\int_{0}^{1} \sigma_{\nu}^{*} \kappa_{D}=\int_{\sigma_{\nu}} \kappa_{D} \leq\left(1+\varepsilon_{\nu}\right) k_{D}\left(z_{\nu}, w_{\nu}\right),
$$

where $\int_{0}^{1} \sigma_{\nu}^{*} \kappa_{D}$ and $\int_{\sigma_{\nu}} \kappa_{D}$ stand for $\int_{0}^{1} \kappa_{D}\left(\sigma_{\nu}(s), \dot{\sigma}_{\nu}(s)\right) d s$. We claim that there exists $\nu_{0}$ such that for every $\nu \geq \nu_{0}$ we have $\sigma_{\nu}([0,1]) \subset V$. If it is not so, then there exist $t_{\nu}=\sigma_{\nu}\left(s_{\nu}\right)$ such that $t_{\nu_{k}} \notin V$ for some subsequence $t_{\nu_{k}}$. We get a contradiction if we show that the sequence $\left\{t_{\nu}\right\}$ converges to $x$. Indeed the sequence $\left\{t_{\nu}\right\}$ verifies the hypotheses of Lemma 1: by the choice of $\varepsilon_{\nu}$ we have

$$
\begin{aligned}
k_{D}\left(z_{\nu}, t_{\nu}\right)+k_{D}\left(t_{\nu}, w_{\nu}\right) & \leq \int_{0}^{s_{\nu}} \sigma_{\nu}^{*} \kappa_{D}+\int_{s_{\nu}}^{1} \sigma_{\nu}^{*} \kappa_{D} \\
& =\int_{0}^{1} \sigma_{\nu}^{*} \kappa_{D} \leq\left(1+\varepsilon_{\nu}\right) k_{D}\left(z_{\nu}, w_{\nu}\right) \leq k_{D}\left(z_{\nu}, w_{\nu}\right)+1 .
\end{aligned}
$$

Therefore, as claimed, we have $\sigma_{\nu}([0,1]) \subset V$, and hence, by (9),

$$
k_{D \cap U}\left(z_{\nu}, t_{\nu}\right) \leq \int_{\sigma_{\nu}} \kappa_{D \cap U} \leq(1+\varepsilon) \int_{\sigma_{\nu}} \kappa_{D} \leq(1+\varepsilon)^{2} k_{D}\left(z_{\nu}, w_{\nu}\right) .
$$

Since $\varepsilon$ and the sequences $\left\{z_{\nu}\right\}$ and $\left\{w_{\nu}\right\}$ are arbitrary, the proof is complete, Q.E.D.

Proposition 4. Let $\Omega \Subset \mathbf{C}^{m}, m \geq n$, be a strongly pseudoconvex domain, let $D^{\prime}$ and $\Omega^{\prime}$ be domains containing respectively $\bar{D}$ and $\bar{\Omega}$ and let $F: D^{\prime} \rightarrow \Omega^{\prime}$ be a proper holomorphic embedding such that $F(D) \subset \Omega, F(\partial D) \subset \partial \Omega$ and such that $F\left(D^{\prime}\right)$ and $\partial \Omega$ are transversal as real submanifolds. Then for every $\varepsilon>0$ there exists a compact subset $K \Subset D$ such that for every choice of $z \in D \backslash K$ and $w \in D$ we have

$$
k_{D}(z, w) \leq(1+\varepsilon) k_{\Omega}(F(z), F(w)) .
$$

Proof. By the transversality assumption there are constants $C_{1}, C_{2}>0$ such that for every $z \in D$

$$
C_{1} d(z, \partial D) \leq d(F(z), \partial \Omega) \leq C_{2} d(z, \partial D) .
$$


Suppose that (10) is not true. Then there exist $\varepsilon_{0}>0$ and two sequences $\left\{z_{\nu}\right\},\left\{w_{\nu}\right\} \subset D$ such that $z_{\nu} \neq w_{\nu}, z_{\nu} \rightarrow \partial D$ and

$$
k_{D}\left(z_{\nu}, w_{\nu}\right) \geq\left(1+\varepsilon_{0}\right) k_{\Omega}\left(F\left(z_{\nu}\right), F\left(w_{\nu}\right)\right) .
$$

We shall prove that

$$
\lim _{\nu \rightarrow+\infty} \frac{k_{D}\left(z_{\nu}, w_{\nu}\right)}{k_{\Omega}\left(F\left(z_{\nu}\right), F\left(w_{\nu}\right)\right)}=1
$$

contradicting (12). By the boundedness of $D$ we can suppose that there exist $x \in \partial D$ and $y \in \bar{D}$ such that $z_{\nu} \rightarrow x$ and $w_{\nu} \rightarrow y$. Suppose that $y \in D$ or $y \in \partial D$ but $x \neq y$. Then, by (5) in the first case and by (6) in the second one, and by (11), we find that for a suitable constant $C$

$$
0 \leq k_{D}\left(z_{\nu}, w_{\nu}\right)-k_{\Omega}\left(F\left(z_{\nu}\right), F\left(w_{\nu}\right)\right) \leq C .
$$

Again by (5) and (6) in both cases we have

$$
\lim _{\nu \rightarrow+\infty} k_{D}\left(z_{\nu}, w_{\nu}\right)=\lim _{\nu \rightarrow+\infty} k_{\Omega}\left(F\left(z_{\nu}\right), F\left(w_{\nu}\right)\right)=+\infty .
$$

By (14) and (15) we immediately obtain (13).

It remains to investigate what happens when $x=y \in \partial D$. In this case, by the transversality assumption and since the domains are strongly pseudoconvex, there are neighborhoods $U$ and $V$ respectively of $x$ and $F(x) \in \partial \Omega$ such that $D \cap U$ and $\Omega \cap V$ are connected, $F(D \cap U) \subset \Omega \cap V$, and there exists a holomorphic retraction $G: \Omega \cap V \rightarrow D \cap U$ for $F$, that is, a mapping such that $G(F(z))=z$ for every $z \in D \cap U$ (cf. [7]). Therefore, by the contraction properties of the Kobayashi distance, the holomorphic mapping $F: D \cap U \rightarrow$ $\Omega \cap V$ is an isometry (for the Kobayashi distance). Thus, by Proposition 3 we get

$$
\lim _{\nu \rightarrow+\infty} \frac{k_{D}\left(z_{\nu}, w_{\nu}\right)}{k_{\Omega}\left(F\left(z_{\nu}\right), F\left(w_{\nu}\right)\right)}=\lim _{\nu \rightarrow+\infty} \frac{k_{D}\left(z_{\nu}, w_{\nu}\right)}{k_{D \cap U}\left(z_{\nu}, w_{\nu}\right)} \frac{k_{\Omega \cap V}\left(F\left(z_{\nu}\right), F\left(w_{\nu}\right)\right)}{k_{\Omega}\left(F\left(z_{\nu}\right), F\left(w_{\nu}\right)\right)}=1,
$$

and the proof is complete, Q.E.D.

Remark. A similar proof can be given of the analogous statement where the Kobayashi distance is replaced by the Kobayashi infinitesimal metric or by the Carathéodory infinitesimal metric.

Now we complete the proof of Theorem 1. Given $D$, by the Fornaess embedding theorem (cf. [3]) there exist a strongly convex bounded domain $\Omega \subset \mathbf{C}^{m}$, $m \geq n$, and a holomorphic embedding $F: D \rightarrow \Omega$ satisfying the hypotheses of Proposition 4. Given $\varepsilon>0$, let $K \Subset D$ be as in (10). Since on convex domains the Kobayashi and Carathéodory distances coincide, using their contraction properties under holomorphic mappings, for every $z \in D \backslash K$ and every $w \in D$ we have

$k_{D}(z, w) \leq(1+\varepsilon) k_{\Omega}(F(z), F(w))=(1+\varepsilon) c_{\Omega}(F(z), F(w)) \leq(1+\varepsilon) c_{D}(z, w)$,

and the proof of Theorem 1 is complete. 
Remark. By Theorem 1 it follows that Propositions 3 and 4 hold also, replacing the Kobayashi distance with the Carathéodory one. It would be interesting to find a proof of Proposition 3 for the Carathéodory distance-and hence of Theorem 1-without using the embedding theorem of Fornaess.

\section{REFERENCES}

1. M. Abate, Boundary behavior of invariant distances and complex geodesics, Rend. Accad. Naz. Lincei 82 (1986), 100-106.

2. A. A. Fadlalla, On boundary value problem in pseudoconvex domains, In Analytic Functions. Błażejewko 1982, Lecture Notes in Math., no. 1039, Springer, Berlin, 1983, 168-176.

3. J. E. Fornaess, Strictly pseudoconvex domains in convex domains, Amer. J. Math. 98 (1976), 529-569.

4. F. Forsternic and J.-P. Rosay, Localization of the Kobayashi metric and the boundary continuity of proper holomorphic mappings, Math. Ann. 279 (1987), 239-252.

5. T. Franzoni and E. Vesentini, Holomorphic maps and invariant distances, Notas Mat., no. 69, North-Holland, Amsterdam, 1980.

6. I. Graham, Boundary behavior of Carathéodory and Kobayashi metrics on strictly pseudoconvex domains in $\mathbf{C}^{n}$ with smooth boundary, Trans. Amer. Math. Soc. 207 (1975), 219-240.

7. G. M. Henkin, Continuation of bounded holomorphic functions from submanifolds in general position in a strictly pseudoconvex domain, Math. USSR-Izv. 6 (1972), 536-563.

8. S. Kobayashi, Hyperbolic manifolds and holomorphic mappings, Dekker, New York, 1970.

9. L. Lempert, La métrique de Kobayashi et la réprésentation des domaines sur la boule, Bull. Soc. Math. France 109 (1981), 427-474.

10. Holomorphic retracts and intrinsic metrics in convex domains, Anal. Math. 8 (1982), 257-261.

11. H. L. Royden, Remarks on the Kobayashi metric, In Several Complex Variables, II, Lecture Notes in Math., no. 189, Springer, Berlin, 1971, pp. 125-137.

12. S. Venturini, Pseudodistances and pseudometrics on real and complex manifolds, Ann. Mat. Pura Appl. (4) (to appear).

13. N. Voormor, Topologische Fortsetzung biholomorphen Funktionen auf dem Rande bei beschränkten streng-pseudokonvexen Gebieten in $\mathbf{C}^{n}$ mit $C^{\infty}$ Rand, Math. Ann. 204 (1973), 239-261.

Scuola Normale Superiore, P.za dei Cavalieri 7, 56100 Pisa, Italy 